\title{
UTILITARIANISM FOR INFINITE UTILITY STREAMS: SUMMABLE DIFFERENCES AND FINITE AVERAGES
}

\author{
ADAM JONSSON AND MARK VOORNEVELD
}

\begin{abstract}
This paper provides axiomatic descriptions of social welfare relations, defined on infinite streams of utility, that are consistent with the utilitarian criterion on subsets where maximizing aggregate utility has a clear interpretation: the streams, or their differences, are summable. Besides standard assumptions on efficiency, equity and interpersonal comparability, two axioms are introduced and shown to be necessary and sufficient. A more general version of one axiom suffices to distinguish streams with different long-run averages.
\end{abstract}

SSE/EFI Working Paper Series in Economics and Finance, No. 747

Date: April 15, 2014.

Key words and phrases. Intergenerational equity; aggregating infinite utility streams; ethical social welfare relations.

We are grateful for detailed comments by José Alcantud, Geir Asheim, Tapan Mitra, Peter Wakker, and Jörgen Weibull. The second author acknowledges financial support by the WallanderHedelius Foundation under grant P2010-0094:1. 


\section{INTRODUCTION}

In the social welfare literature on infinite utility streams, there is a long tradition of combining two basic desiderata: the Strong Pareto SP axiom requires social preferences to reflect Pareto improvements and Finite Anonymity FA ensures equal treatment of generations by imposing invariance to transformations that swap the utility levels of two (or, by transitivity, finitely many) generations; precise definitions are in Section 3. We study intuitive infinite-horizon generalizations of classical utilitarianism for finitely many generations in combination with these two minimal requirements of equity and efficiency. The well-known results of Basu and Mitra [3], Zame [21] and Lauwers [12] tell us that no complete ordering satisfying the two requirements can be represented by a social welfare function or otherwise be described explicitly. This is an inconvenient state of affairs for policy analysis and, more generally, for transparent decision making, as one would like to have explicit criteria for evaluating streams of utility. Nevertheless, their results imply that some streams will inevitably be noncomparable.

Faced with these restrictions, we study incomplete social preferences that satisfy intuitive generalizations of finite utilitarianism to an infinite horizon setting. For a finite number $n$ of generations, classical utilitarianism (see Moulin [15, p. 21] for a textbook treatment) declares utility profile $u=\left(u_{1}, \ldots, u_{n}\right)$ to be weakly preferred to $v=\left(v_{1}, \ldots, v_{n}\right)$, written $u \succsim v$, if and only if

$$
\sum_{i=1}^{n} u_{i} \geq \sum_{i=1}^{n} v_{i}
$$

For a countably infinite number of future generations, while the explicit construction of complete preferences is constrained by the theorems of Lauwers [12] and Zame [21], an obvious extension of (1) would require

$$
u \succsim v \text { if } u, v \text { have finite sums with } \sum_{i=1}^{\infty} u_{i} \geq \sum_{i=1}^{\infty} v_{i}
$$

or, more generally,

$$
\begin{aligned}
& u \succsim v \text { for streams } u, v \text { whose difference } u-v \text { is summable } \\
& \text { and satisfies } \sum_{i=1}^{\infty}\left(u_{i}-v_{i}\right) \geq 0 .
\end{aligned}
$$

Banerjee [1, p. 329], for instance, explicitly expresses the desirability of (2).

Our aim is to characterize when a social welfare relation (SWR; a reflexive, transitive binary relation) or an ethical SWR (a SWR satisfying SP and FA) satisfies (2) or (3). Besides a standard axiom on interpersonal comparison of utilities, we introduce two new axioms. The first axiom, Horizon Consistency HC, expresses a consistency between large, finite-horizon problems and the infinite-horizon problem, much in the spirit of the consistency requirement in Brock's [5] classical characterization of von Weizsäcker's [19] overtaking criterion. The second axiom is a mild continuity requirement.

Our main results are summarized as follows: Theorems 1 to 4 all provide characterizations of relations satisfying (2) or (3) on two domains that appear frequently in the literature. Logical independence of the axioms is discussed in section 4.2. Our characterizations of SWRs satisfying (2) and (3) answer an open question from 
Banerjee [1, p. 335] whether such relations "can be characterized without postulating any form of continuity": our results show that a certain continuity condition (in our notation, $\left.\varepsilon \mathrm{C}\left(\mathscr{U}_{\mathrm{fin}}\right)\right)$ is indeed necessary for utilitarian requirements (2) and (3). Finally, Theorem 5 shows that imposing some of our axioms on streams with welldefined, finite averages gives sufficient conditions for SWRs to discriminate among streams with distinct averages and Theorem 6 provides a new characterization of the utilitarian SWR of Basu and Mitra [4]; the latter is done on a larger domain and under a weaker assumption concerning interpersonal comparison of utilities.

To our knowledge, a careful examination of necessary and sufficient conditions for utilitarian condition (2) on streams that are summable or (3) on streams with a summable difference has not been conducted before. There are several characterizations of utilitarianism in finite-horizon problems, including Milnor [14], Maskin [13], and d'Aspremont and Gevers [7]. ${ }^{1}$ Basu and Mitra adapt the latter to infinite utility streams to characterize a utilitarian social welfare relation (defined in (5) below) that leads to rankings that are easily accepted, but only allows comparison of utility streams where one dominates the other beyond some finite horizon: their utilitarian SWR does not satisfy the intuitive generalizations of utilitarianism in (2) and (3). ${ }^{2}$ They ask the general question which additional continuity properties can be motivated by ethical principles in this context (cf. [4, p. 357]). As indicated above, Banerjee [1] explicitly raises the particular issue that we discuss. His focus, however, is on extending the utilitarian SWR of [4] and the Suppes-Sen grading principle ([17], [16]) to allow for extended anonymity conditions using classes of infinite permutations. He does not address the characterization of SWRs satisfying (2) or (3).

\section{Notation}

$\mathbb{N}=\{1,2,3, \ldots\}$ is the set of positive integers, $\mathbb{R}$ the set of real numbers,

$$
\mathscr{U}=\left\{u \in \mathbb{R}^{\mathbb{N}}: \sup _{n \in \mathbb{N}}\left|u_{n}\right|<+\infty\right\}
$$

is the set of (bounded) utility streams $u=\left(u_{1}, u_{2}, \ldots\right)$, where $u_{n}$ represents the welfare level of generation $n \in \mathbb{N}$. Our axioms are sometimes indexed by subsets $\mathscr{V}$ of $\mathscr{U}$. The three most important instances of subsets $\mathscr{V}$ are the set of streams that are eventually zero, summable, and have well-defined (necessarily finite) averages,

\footnotetext{
${ }^{1}$ Less directly related are papers in utility theory by Wakker [20], who restricts attention to streams that are eventually equal to a given constant, and Fishburn and Edwards [8], who only compare streams that differ in finitely many coordinates.

${ }^{2}$ Tapan Mitra (private communication) mentioned that they were aware of this shortcoming of their utilitarian SWR; in a short uncirculated note of February 2004, he had proposed another relation: for all streams $u, v$, (a) if $u-v$ is summable, $u \succsim v$ iff $\sum_{i=1}^{\infty}\left(u_{i}-v_{i}\right) \geq 0$; (b) if $u-v$ is not summable, $u \succsim v$ iff there is an $n \in \mathbb{N}$ with $\sum_{i=1}^{n} u_{i} \geq \sum_{i=1}^{n} v_{i}$ and $u_{i} \geq v_{i}$ for all $i>n$. This binary relation satisfies (2) and (3) by property (a), but is not transitive. See the discussion following Theorem 3 for further examples.
} 
respectively:

$$
\begin{aligned}
\mathscr{U}_{0} & =\left\{u \in \mathscr{U}: u_{n}=0 \text { for all but finitely many } n \in \mathbb{N}\right\}, \\
\mathscr{U}_{\text {fin }} & =\left\{u \in \mathscr{U}: \sum_{n=1}^{\infty} u_{n}=\lim _{N \rightarrow \infty} \sum_{n=1}^{N} u_{n} \text { exists in } \mathbb{R}\right\}, \\
\mathscr{U}_{\text {av }} & =\left\{u \in \mathscr{U}: \bar{u}=\lim _{n \rightarrow \infty}\left(u_{1}+\cdots+u_{n}\right) / n \text { exists in } \mathbb{R}\right\} .
\end{aligned}
$$

Note that $\mathscr{U}_{0} \subset \mathscr{U}_{\text {fin }} \subset \mathscr{U}_{\text {av }} \subset \mathscr{U}$.

Given $u, v \in \mathscr{U}$, we write $u \geq v$ and say that $u$ dominates $v$ if $u_{n} \geq v_{n}$ for all $n$. We write $u>v$ if $u \geq v$ and $u \neq v$. For $u \in \mathscr{U}_{\text {fin }}$, let

$$
\sigma(u):=\sum_{i=1}^{\infty} u_{i}
$$

For $j \in \mathbb{N}, e_{j}$ is the stream with $j$-th coordinate equal to one and all others equal to zero. For $n \in \mathbb{N}, c=\left(c_{1}, \ldots, c_{n}\right) \in \mathbb{R}^{n}, a \in \mathbb{R}$ and $u \in \mathscr{U}$, we sometimes write

$$
\begin{aligned}
& \underline{a}_{n}=(\overbrace{a, \ldots, a}^{n \text { times }}), \quad \underline{a}=(a, a, \ldots), \quad a u=\left(a u_{1}, a u_{2}, \ldots\right), \\
& \left(c_{1}, \ldots, c_{n}\right)^{\infty}=\left(c_{1}, \ldots, c_{n}, c_{1}, \ldots, c_{n}, c_{1}, \ldots\right), \\
& (c, u)=\left(c_{1}, \ldots, c_{n}, u_{1}, u_{2}, \ldots\right), \\
& u_{[n]}=\left(u_{1}, \ldots, u_{n}, \underline{0}\right), \quad u^{[n]}=\left(u_{n+1}, u_{n+2}, \ldots\right) .
\end{aligned}
$$

A social welfare relation (SWR) is a reflexive and transitive binary relation $\succsim$ on $\mathscr{U} ; u \succsim v$ means that $u$ is considered at least as good as $v$ by society, $u \sim v$ means that $u \succsim v$ and $v \succsim u, u \succ v$ means that $u \succsim v$ holds but $v \succsim u$ does not. We call $u$ and $v(\succsim$-) comparable if $u \succsim v$ or $v \succsim u$, or both. The SWR $\succsim$ is an extension of the SWR $\succsim^{\prime}$ if for all $u, v \in \mathscr{U}:$ (i) $u \succsim^{\prime} v$ implies $u \succsim v$ and (ii) $u \succ^{\prime} v$ implies $u \succ v$. If $\succsim$ extends $\succsim^{\prime}$, we call $\succsim^{\prime}$ weaker than $\succsim$.

\section{Axioms}

An ethical (some authors use the term equitable) SWR, or ESWR for short, is a social welfare relation that satisfies the Strong Pareto and Finite anonymity axioms:

Strong Pareto, SP: For all $u, v \in \mathscr{U}$, if $u>v$, then $u \succ v$.

Finite anonymity, FA: For all $u, v \in \mathscr{U}$, if there are $i, j \in \mathbb{N}$ with $u_{i}=v_{j}, u_{j}=v_{i}$, and $u_{k}=v_{k}$ for all other $k \in \mathbb{N} \backslash\{i, j\}$, then $u \sim v$.

Finite-dimensional utilitarianism (1) is characterized by SP, FA, and a further assumption on interpersonal comparability of utility; see, for example, d'Aspremont and Gevers [7]. We state two versions of interpersonal comparability for the infinite horizon context:

Unit comparability, UC: For all $u, v \in \mathscr{U}$, if $u \succsim v$, then $u+\alpha \succsim$ $v+\alpha$ for every $\alpha \in \mathscr{U}$.

Finite unit comparability, FC: For all $u, v \in \mathscr{U}$, if $u \succsim v$, then $u+\alpha \succsim v+\alpha$ for every $\alpha \in \mathscr{U}_{0}$.

If a SWR $\succsim$ is defined in terms of the difference $u-v$ of the streams which are to be compared, then $u-v=(u+\alpha)-(v+\alpha)$ for all $\alpha \in \mathscr{U}$. So if $\succsim$ satisfies FC, then it also satisfies UC. This is the case with, for instance, Basu and Mitra's 
[4] utilitarian criterion, Gale's [9] formulations of von Weizsäcker's [19] overtaking criterion and the catching-up criterion, and Jonsson and Voorneveld's [10] limitdiscounted utilitarian criterion; for definitions, see (5), (6), (10), and (11) below.

The following axioms will be used in the main characterization:

Horizon consistency, HC: For all $u, v \in \mathscr{U}$, if there is an $N \in \mathbb{N}$ with $u_{[n]} \succ v_{[n]}$ for all $n \geq N$, then $u \succsim v$.

Epsilon-continuity, $\varepsilon \mathrm{C}$ : For all $u, v \in \mathscr{U}$, if $u+(\varepsilon, \underline{0}) \succsim v$ for all $\varepsilon>0$, then $u \succsim v$.

Epsilon-continuity appears to be original to our approach. Using perturbations of the first (or, in combination with Finite Anonymity, a single) coordinate only, it is an extremely weak continuity requirement. For instance, $u+(\varepsilon, \underline{0})$ converges to $u$ as $\varepsilon$ goes to zero in all but one of the standard topologies on $\mathscr{U}$ in [11, Sec. 3] and [2, Sec. 2.3; their properties M.1 and M.4 of a metric suffice for this conclusion]; the only exception is the topology of Campbell [6] generated by metric $d(u, v)=\sup \left\{\delta\left(u_{n}, v_{n}\right) / n: n \in \mathbb{N}\right\}$, with $\delta\left(u_{n}, v_{n}\right)=0$ if $u_{n}=v_{n}$ and $\delta\left(u_{n}, v_{n}\right)=1$ otherwise. So it is implied by upper semicontinuity (closed upper contour sets $U(v)=\{u \in \mathscr{U}: u \succsim v\}$ for each $v$ ) in all these topologies. ${ }^{3}$ Horizon Consistency captures the intuition formulated in Brock [5, p. 929] that "decisions on infinite programs are consistent with decisions on finite programs of length $n$ if $n$ is large enough". However, $\mathrm{HC}$ is a weaker variant of Brock's third axiom (and of the related "weak consistency" axiom in [4, p. 359]), which requires that $u \succ v$ holds if $u_{[n]} \succ v_{[n]}$ for large $n$. This stronger form of $\mathrm{HC}$ does not allow us to order summable streams by their sums. Indeed, it is not hard to verify that if SWR $\succsim$ satisfies the stronger form of $\mathrm{HC}$ and

$$
\text { for all } u, v \in \mathscr{U}_{0}: \quad u \succsim v \quad \Leftrightarrow \quad \sigma(u) \geq \sigma(v),
$$

then $\succsim$ must rank $u=(1,0,0,0, \ldots)$ above $v=(1 / 2,1 / 4,1 / 8,1 / 16, \ldots)$. Horizon Consistency on the other hand is compatible with (2) and (3); the restriction of $\mathrm{HC}$ to $\mathscr{U}_{\text {fin }}$ is a necessary condition (Theorems $1,2,3$ ).

Each axiom above can be restricted to an arbitrary subset $\mathscr{V}$ of $\mathscr{U}$ by requiring only that the condition in question hold when $u$ and $v$ are both in $\mathscr{V}$. The axioms thus obtained are denoted $\mathrm{SP}(\mathscr{V}), \mathrm{FA}(\mathscr{V}), \mathrm{UC}(\mathscr{V}), \mathrm{FC}(\mathscr{V}), \mathrm{HC}(\mathscr{V})$ and $\varepsilon \mathrm{C}(\mathscr{V})$. When $\mathscr{V}=\mathscr{U}$, the index set will be omitted.

We say that a SWR $\succsim$ on $\mathscr{U}$ orders streams with finite sums by their sums if

$$
\forall u, v \in \mathscr{U}_{\text {fin }}: u \succsim v \quad \Leftrightarrow \quad \sum_{n=1}^{\infty} u_{n} \geq \sum_{n=1}^{\infty} v_{n} .
$$

For ethical SWRs, Theorem 1 shows that $\mathrm{FC}\left(\mathscr{U}_{0}\right), \mathrm{HC}\left(\mathscr{U}_{\text {fin }}\right)$ and $\varepsilon \mathrm{C}\left(\mathscr{U}_{\text {fin }}\right)$ are necessary and sufficient conditions for (4), so it may be worthwhile discussing them in relation to two ESWRs that do not satisfy (4). We denote the utilitarian SWR of Basu and Mitra [4] and von Weizsäcker's overtaking criterion as formalized in

${ }^{3} \mathrm{~A}$ slightly weaker version, $\mathrm{W} \varepsilon \mathrm{C}$, suffices in our proofs:

$$
\text { for all } u, v \in \mathscr{U} \text {, if } u+(\varepsilon, \underline{0}) \succ v \text { for all } \varepsilon>0 \text {, then } u \succsim v
$$

But the Pareto axiom appearing in all our theorems makes the two versions equivalent. For the nontrivial direction, let SWR $\succsim$ satisfy SP and $\mathrm{W} \varepsilon \mathrm{C}$ and let $u, v \in \mathscr{U}$ have $u+(\varepsilon, \underline{0}) \succsim v$ for all $\varepsilon>0$. For all $\varepsilon>0$, SP and $\mathrm{W} \varepsilon \mathrm{C}$ give $u+(\varepsilon, \underline{0}) \succ u+(\varepsilon / 2, \underline{0}) \succsim v$, so $u+(\varepsilon, \underline{0}) \succ v$ and $u \succsim v$ by $\mathrm{W} \varepsilon \mathrm{C}$. 
Svensson [18] and Gale [9] by $\succsim_{U}$ and $\succsim_{W}$, respectively:

$$
\begin{aligned}
& u \succsim_{U} v \Leftrightarrow \exists N \in \mathbb{N} \text { with } \sum_{i=1}^{N}\left(u_{i}-v_{i}\right) \geq 0 \text { and } u_{n}-v_{n} \geq 0 \text { for all } n>N, \\
& u \succsim_{W} v \Leftrightarrow \exists N \in \mathbb{N} \text { with } \sum_{i=1}^{n}\left(u_{i}-v_{i}\right) \geq 0 \text { for all } n \geq N .
\end{aligned}
$$

To see that $\succsim_{U}$ violates $\mathrm{HC}\left(\mathscr{U}_{\text {fin }}\right)$, take $u=(1 / 2,1 / 4,1 / 8, \ldots)$ and $v=(1,0,0, \ldots)$. Then $v_{[n]} \succ_{U} u_{[n]}$ for every $n$, but there is no $N \in \mathbb{N}$ with $\sum_{n=1}^{N}\left(v_{n}-u_{n}\right) \geq 0: u$ and $v$ are not $\succsim_{U}$-comparable. It violates $\varepsilon \mathrm{C}$ as well: $u+(\varepsilon, \underline{0}) \succsim_{U} v$ for all $\varepsilon>0$, but $u$ and $v$ are not $\succsim_{U}$-comparable. The overtaking SWR satisfies FC and (the above mentioned stronger form of $) \mathrm{HC}$. That $\varepsilon \mathrm{C}\left(\mathscr{U}_{\text {fin }}\right)$ fails can be seen by noting that, for the same $u$ and $v$, we have $v \succ_{W} u$ even though $u+(\varepsilon, \underline{0}) \succsim_{W} v$ for every $\varepsilon>0$.

\section{MAin Results}

\subsection{Finite sums.}

Theorem 1. An ESWR $\succsim$ on $\mathscr{U}$ orders streams with finite sums by their sums if and only if it satisfies $\mathrm{FC}\left(\mathscr{U}_{0}\right), \mathrm{HC}\left(\mathscr{U}_{\text {fin }}\right)$ and $\varepsilon \mathrm{C}\left(\mathscr{U}_{\text {fin }}\right)$.

We start with a lemma for the set $\mathscr{U}_{0}$.

Lemma 1. Let $\succsim$ be a $S W R$ on $\mathscr{U}$.

(a) If $\succsim$ satisfies $\mathrm{FA}$ and $\mathrm{FC}$ on $\mathscr{U}_{0}$, then for all $u \in \mathscr{U}_{0}: u \sim(\sigma(u), \underline{0})$.

(b) If $\succsim$ satisfies $\mathrm{FA}, \mathrm{SP}$, and $\mathrm{FC}$ on $\mathscr{U}_{0}$, then for all $u, v \in \mathscr{U}_{0}: u \succsim v$ if and only if $\sigma(u) \geq \sigma(v)$.

Proof. (a): We first prove that

$$
\text { for all } j \in \mathbb{N}, a \in \mathbb{R}: \quad a e_{1}-a e_{j} \sim \underline{0} .
$$

If $j=1$, this follows from reflexivity of $\succsim$. So let $j>1$. By $\mathrm{FA}\left(\mathscr{U}_{0}\right)$,

$$
\frac{1}{2} a e_{1}-\frac{1}{2} a e_{j} \sim-\frac{1}{2} a e_{1}+\frac{1}{2} a e_{j} .
$$

By $\operatorname{FC}\left(\mathscr{U}_{0}\right)$ with $\alpha=\frac{1}{2} a e_{1}-\frac{1}{2} a e_{j}$, this gives (7).

For $u \in \mathscr{U}_{0}$, let $m(u)=\min \left\{n \in \mathbb{N}: u_{k}=0\right.$ for all $\left.k>n\right\}$. If $m(u)=1$, $u=(\sigma(u), \underline{0})$, so $u \sim(\sigma(u), \underline{0})$ by reflexivity of $\succsim$. If $m(u)=n>1$, (7) gives $u_{n} e_{1}-u_{n} e_{n} \sim \underline{0}$, so by $\mathrm{FC}\left(\mathscr{U}_{0}\right)$, adding $u$ to both sides gives

$$
\left(u_{1}+u_{n}, u_{2}, \ldots, u_{n-1}, \underline{0}\right) \sim u .
$$

The left stream has at most $n-1$ nonzero coordinates. Repeating with $u_{n-1} e_{1}-$ $u_{n-1} e_{n-1} \sim \underline{0}, \ldots, u_{2} e_{1}-u_{2} e_{2} \sim \underline{0}$, each time changing a (possibly) nonzero coordinate to zero and adding it to coordinate 1 , we find that $u \sim(\sigma(u), \underline{0})$.

(b): Let $u, v \in \mathscr{U}_{0}$. By (a), $u \succsim v$ if and only if $(\sigma(u), \underline{0}) \succsim(\sigma(v), \underline{0})$. By reflexivity of $\succsim$ if $\sigma(u)=\sigma(v)$ and Pareto axiom $\operatorname{SP}\left(\mathscr{U}_{0}\right)$ otherwise, the latter is equivalent with $\sigma(u) \geq \sigma(v)$.

This leaves us properly equipped for the proof of Theorem 1: 
Proof. First, let ESWR $\succsim$ satisfy (4). It clearly satisfies FC on $\mathscr{U}_{0}$. For $\varepsilon \mathrm{C}$ on $\mathscr{U}_{\text {fin }}$, let $u, v \in \mathscr{U}_{\text {fin }}$ have $u+(\varepsilon, \underline{0}) \succsim v$ for every $\varepsilon>0$. By (4), this is equivalent with $\sum_{n=1}^{\infty} u_{n}+\varepsilon \geq \sum_{n=1}^{\infty} v_{n}$ for every $\varepsilon>0$. This implies $\sum_{n=1}^{\infty} u_{i} \geq \sum_{n=1}^{\infty} v_{i}$ and, by (4), that $u \succsim v$. For HC on $\mathscr{U}_{\text {fin }}$, let $u, v \in \mathscr{U}_{\text {fin }}$ and $N \in \mathbb{N}$ be such that $u_{[n]} \succ v_{[n]}$ for all $n \geq N$. By (4), this means that $\sum_{i=1}^{n} u_{i}>\sum_{i=1}^{n} v_{i}$ for all $n \geq N$. Hence, $\sum_{n=1}^{\infty} u_{i} \geq \sum_{n=1}^{\infty} v_{i}$ and, by (4), $u \succsim v$.

Conversely, let ESWR $\succsim$ satisfy the axioms. As $\mathscr{U}_{0} \subset \mathscr{U}_{\text {fin }}$, Lemma 1 applies. Let $u, v \in \mathscr{U}_{\text {fin }}$.

If $\sum_{n=1}^{\infty} u_{n} \geq \sum_{n=1}^{\infty} v_{n}$, let $\varepsilon>0$. Since the two series converge, there is an $N \in \mathbb{N}$ with $\sum_{i=1}^{n} u_{i}+\frac{\varepsilon}{2}>\sum_{i=1}^{n} v_{i}$ for all $n \geq N$. Equivalently: for all $n \geq N$, the streams $u_{[n]}+(\varepsilon / 2, \underline{0})$ and $v_{[n]}$ satisfy

$$
\sigma\left(u_{[n]}+(\varepsilon / 2, \underline{0})\right)=\sum_{i=1}^{n} u_{i}+\frac{\varepsilon}{2}>\sum_{i=1}^{n} v_{i}=\sigma\left(v_{[n]}\right) .
$$

By Lemma $1(\mathrm{~b}), u_{[n]}+(\varepsilon / 2, \underline{0}) \succ v_{[n]}$ for all $n \geq N$. By $\operatorname{HC}\left(\mathscr{U}_{\text {fin }}\right), u+(\varepsilon / 2, \underline{0}) \succsim v$. By SP, $u+(\varepsilon, \underline{0}) \succ v$. Since $\varepsilon>0$ was arbitrary, $\varepsilon \mathrm{C}\left(\mathscr{U}_{\text {fin }}\right)$ gives $u \succsim v$.

If $u \succsim v$, we show that $\sum_{n=1}^{\infty} u_{n} \geq \sum_{n=1}^{\infty} v_{n}$. If, to the contrary, $\sum_{n=1}^{\infty} u_{n}<$ $\sum_{n=1}^{\infty} v_{n}$, then $\sum_{n=1}^{\infty} v_{n}-\varepsilon \geq \sum_{n=1}^{\infty} u_{n}$ for $\varepsilon>0$ sufficiently small. Applying the previously proved step to summable $v-(\varepsilon, \underline{0})$ and $u$ gives $v-(\varepsilon, \underline{0}) \succsim u$. By SP, $v \succ u$, contradicting $u \succsim v$.

Careful reading of the proof of Theorem 1 reveals that the unrestricted forms of Strong Pareto and Finite anonymity are unnecessarily strong for conclusion (4): FA is used only on $\mathscr{U}_{0}$ and SP is used only on $\mathscr{U}_{\text {fin }}$. Conversely, (4) clearly implies FA on $\mathscr{U}_{0}$ and SP on $\mathscr{U}_{\text {fin }}$. This gives the following generalization of Theorem 1:

Theorem 2. A $S W R \succsim$ on $\mathscr{U}$ orders streams with finite sums by their sums if and only if it satisfies $\mathrm{FA}\left(\mathscr{U}_{0}\right), \mathrm{FC}\left(\mathscr{U}_{0}\right), \mathrm{SP}\left(\mathscr{U}_{\text {fin }}\right), \mathrm{HC}\left(\mathscr{U}_{\text {fin }}\right)$, and $\varepsilon \mathrm{C}\left(\mathscr{U}_{\text {fin }}\right)$.

Theorem 3. A social welfare relation $\succsim$ on $\mathscr{U}$ satisfies

$$
\text { for all } u, v \in \mathscr{U} \text { with } u-v \in \mathscr{U}_{\text {fin }}: \quad u \succsim v \quad \Leftrightarrow \quad \sum_{n=1}^{\infty}\left(u_{n}-v_{n}\right) \geq 0,
$$

if and only if it satisfies $\mathrm{FA}\left(\mathscr{U}_{0}\right), \mathrm{SP}\left(\mathscr{U}_{\text {fin }}\right), \mathrm{HC}\left(\mathscr{U}_{\text {fin }}\right), \varepsilon \mathrm{C}\left(\mathscr{U}_{\text {fin }}\right)$ and the following extension of the Finite Unit Comparability axiom:

for all $u, v \in \mathscr{U}$ with $u-v \in \mathscr{U}_{\text {fin }}$ and all $\alpha \in \mathscr{U}: u \succsim v$ implies $u+\alpha \succsim v+\alpha$.

Proof. That a SWR $\succsim$ satisfying (8) also satisfies the axioms is shown analogously as before; in the interest of brevity, we omit it. Conversely, let SWR $\succsim$ satisfy the axioms. Let $u, v \in \mathscr{U}$ have $u-v \in \mathscr{U}_{\text {fin }}$. Applying the extended unit comparability property (9) twice (once for each direction), we see that $u \succsim v$ if and only if $u-v \succsim \underline{0}$. The extended unit comparability property implies finite unit comparability on $\mathscr{U}_{0}$, so Theorem 2 applies. Since $u-v$ and $\underline{0}$ lie in $\mathscr{U}_{\text {fin }}$, this theorem gives that $u-v \succsim \underline{0}$ if and only if $\sum_{n=1}^{\infty}\left(u_{n}-v_{n}\right) \geq \sum_{n=1}^{\infty} \underline{0}_{n}=0$.

In Section 3, we saw that the utilitarian SWR $\succsim_{U}$ and the overtaking criterion $\succsim_{W}$ violate some of the properties in the preceding theorems and therefore do not order all summable streams according to their sums. Two examples of ethical SWRs that do satisfy all properties in the preceding theorems (as well as those in Theorem 


\begin{tabular}{cccccc} 
& $\mathrm{FA}\left(\mathscr{U}_{0}\right)$ & $\mathrm{FC}\left(\mathscr{U}_{0}\right)$ & $\mathrm{SP}\left(\mathscr{U}_{\text {fin }}\right)$ & $\mathrm{HC}\left(\mathscr{U}_{\text {fin }}\right)$ & $\varepsilon \mathrm{C}\left(\mathscr{U}_{\text {fin }}\right)$ \\
\cline { 2 - 6 }$\succsim_{W}$ & + & + & + & + & - \\
$\succsim_{1}$ & + & + & + & - & + \\
$\succsim_{2}$ & + & + & - & + & + \\
$\succsim_{3}$ & + & - & + & + & + \\
$\succsim_{4}$ & - & + & + & + & + \\
\cline { 2 - 6 } & & & & &
\end{tabular}

TABLE 1 . SWRs and the axioms they do $(+)$ or do not (-) satisfy.

5) and consequently do satisfy the intuitive utilitarian requirements (2) and (3) are the catching-up criterion of Gale [9, p. 3]:

$$
\text { for all } u, v \in \mathscr{U}: \quad u \succsim v \Leftrightarrow \liminf _{n \rightarrow \infty} \sum_{i=1}^{n}\left(u_{i}-v_{i}\right) \geq 0,
$$

and the limit-discounted utilitarian criterion of Jonsson and Voorneveld [10]:

$$
\text { for all } u, v \in \mathscr{U}: \quad u \succsim v \Leftrightarrow \liminf _{\delta \rightarrow 1^{-}} \sum_{i=1}^{\infty} \delta^{i-1}\left(u_{i}-v_{i}\right) \geq 0 .
$$

4.2. Logical independence. The axioms in each of the preceding theorems 1,2 , and 3 are logically independent. We show this for the five axioms $\mathrm{FA}\left(\mathscr{U}_{0}\right), \mathrm{FC}\left(\mathscr{U}_{0}\right)$, $\mathrm{SP}\left(\mathscr{U}_{\text {fin }}\right), \mathrm{HC}\left(\mathscr{U}_{\text {fin }}\right)$, and $\varepsilon \mathrm{C}\left(\mathscr{U}_{\text {fin }}\right)$ in Theorem 2 by providing five SWRs, each violating exactly one of those axioms. The same relations can be used to establish logical independence in the other theorems. Verifying that a SWR satisfies the given properties is routine work (available upon request); we only show explicitly which axiom is violated. Consider the overtaking criterion $\succsim_{W}$ defined in (6) and the four SWRs defined as follows:

$$
\begin{aligned}
& u \succsim_{1} v \Leftrightarrow \exists N \in \mathbb{N} \text { with } u^{[N]} \geq v^{[N]} \text { and } \liminf _{n \rightarrow \infty} \sum_{i=1}^{n}\left(u_{i}-v_{i}\right) \geq 0, \\
& u \succsim_{2} v \text { for all } u, v \quad \text { (i.e., under } \succsim_{2} \text {, all streams are equivalent), } \\
& u \succsim_{3} v \Leftrightarrow \liminf _{n \rightarrow \infty} \sum_{i=1}^{n}\left(u_{i}^{3}-v_{n}^{3}\right) \geq 0, \\
& u \succsim_{4} v \Leftrightarrow u \geq v .
\end{aligned}
$$

The lower limits are defined in $\mathbb{R} \cup\{-\infty,+\infty\}$. Properties are summarized in Table 1. We discussed those of $\succsim_{W}$ after (6).

$\succsim_{1}$ violates $\mathrm{HC}\left(\mathscr{U}_{\text {fin }}\right)$ : take $u=(1 / 2,0,1 / 4,0,1 / 8,0,1 / 16,0, \ldots)$ obtained by taking powers of $1 / 2$ followed by a zero. Similarly, take $v=(0,1 / 4,0,1 / 8,0,1 / 16, \ldots)$. Then $u_{[n]} \succ_{1} v_{[n]}$ for all $n$, but $u$ and $v$ are not $\succsim_{1}$-comparable.

$\succsim_{2}$ violates $\operatorname{SP}\left(\mathscr{U}_{\text {fin }}\right):(2, \underline{0})>(1, \underline{0})$, but $(2, \underline{0}) \sim_{2}(1, \underline{0})$. $\succsim_{3}$ violates $\mathrm{FC}\left(\mathscr{U}_{0}\right)$ : take $u=(0.5, \underline{0}), v=(0.4,0.4, \underline{0}), \alpha=(0.1, \underline{0})$. Then $\sum_{n=1}^{\infty}\left(u_{n}^{3}-v_{n}^{3}\right)=-0.003$, so $v \succ_{3} u$, but $\sum_{n=1}^{\infty}\left(\left(u_{n}+\alpha_{n}\right)^{3}-\left(v_{n}+\alpha_{n}\right)^{3}\right)=0.027$, so $u+\alpha \succ_{3} v+\alpha$.

$\succsim_{4}$ violates $\mathrm{FA}\left(\mathscr{U}_{0}\right): e_{2}$ is obtained from $e_{1}$ by permuting the first two coordinates, but $e_{1}$ and $e_{2}$ are not $\succsim_{4}$-comparable. 
Remark 1. For each of the five SWRs, when an axiom is satisfied, it is satisfied on $\mathscr{U}$. (An axiom that fails on a subset automatically fails on the full domain.) So these $S W R$ s also show that $\mathrm{FA}, \mathrm{FC}, \mathrm{SP}, \mathrm{HC}$, and $\varepsilon \mathrm{C}$ are logically independent.

4.3. The domain $[0,1]^{\mathbb{N}}$. Some papers take the domain of utility streams to be the set $[0,1]^{\mathbb{N}}$ of sequences in $[0,1]$. We stated the results above on the larger set $\mathscr{U}$ of bounded streams. Firstly, this is less restrictive. Secondly, this set is closed under addition and scalar multiplication, which makes many of our axioms easy to state; on $[0,1]^{\mathbb{N}}$, one often needs the caveat that all manipulated streams belong to that domain as well. For instance, the appropriate modifications of Epsilon-Continuity and Finite Unit Comparability on subsets of $[0,1]^{\mathbb{N}}$ are as follows:

Finite unit comparability $\mathrm{FC}$ on $[0,1]^{\mathbb{N}} \cap \mathscr{U}_{0}$ : For all $u, v \in[0,1]^{\mathbb{N}} \cap$ $\mathscr{U}_{0}$ and all $\alpha \in \mathbb{R}^{\mathbb{N}} \cap \mathscr{U}_{0}$ such that $u+\alpha$ and $v+\alpha$ belong to $[0,1]^{\mathbb{N}}$ : if $u \succsim v$, then also $u+\alpha \succsim v+\alpha$.

Epsilon-continuity $\varepsilon \mathrm{C}$ on $[0,1]^{\mathbb{N}} \cap \mathscr{U}_{\text {fin }}$ : For all $u, v \in[0,1]^{\mathbb{N}} \cap \mathscr{U}_{\text {fin }}$, if $u_{1}<1$ and $u+(\varepsilon, \underline{0}) \succsim v$ for all $\varepsilon>0$ with $u+(\varepsilon, \underline{0}) \in[0,1]^{\mathbb{N}}$, then $u \succsim v$.

With appropriate modifications, however, our results remain valid:

Theorem 4. Let $\succsim$ be a $S W R$ on $[0,1]^{\mathbb{N}}$.

(a) Let $\succsim$ satisfy $\mathrm{FA}$ and $\mathrm{FC}$ on $[0,1]^{\mathbb{N}} \cap \mathscr{U}_{0}$. If $u \in[0,1]^{\mathbb{N}}$ has $u_{n}=0$ for all $n$ greater than or equal to some $N \in \mathbb{N}$, then

$$
\text { for all } n \geq N: \quad u \sim(\underbrace{\sigma(u) / n, \ldots, \sigma(u) / n}_{n \text { coord }}, \underline{0}) \in[0,1]^{\mathbb{N}} .
$$

(b) If $\succsim$ satisfies $\mathrm{FA}, \mathrm{SP}$, and $\mathrm{FC}$ on $[0,1]^{\mathbb{N}} \cap \mathscr{U}_{0}$, then for all $u, v \in[0,1]^{\mathbb{N}} \cap \mathscr{U}_{0}$, $u \succsim v$ if and only if $\sigma(u) \geq \sigma(v)$.

(c) $\succsim$ orders streams in $[0,1]^{\mathbb{N}} \cap \mathscr{U}_{\text {fin }}$ by their sums if and only if it satisfies $\mathrm{FC}$ on $[0,1]^{\mathbb{N}} \cap \mathscr{U}_{0}$ and $\mathrm{FA}, \mathrm{SP}, \mathrm{HC}$, and $\varepsilon \mathrm{C}$ on $[0,1]^{\mathbb{N}} \cap \mathscr{U}_{\mathrm{fin}}$.

Proof. (a): This follows from Lemma 1(i) of [4], which states that $u \sim v$ for all $u, v \in[0,1]^{\mathbb{N}}$ with at most finitely many elements distinct from zero. They use a slightly different unit comparability axiom for streams $u, v$ with $u_{n}=v_{n}$ for all large $n$ (see (13) below), where we more specifically assume that $u_{n}=v_{n}=0$ for all large $n$. But all streams in their proof of Lemma 1(i) are eventually zero, so the distinction doesn't matter for our purposes. ${ }^{4}$

(b): Let $u, v \in[0,1]^{\mathbb{N}}$ both have $u_{n}=v_{n}=0$ for sufficiently large $n$. Picking such an $n$ and using (12), $u \succsim v$ if and only if

$$
(\sigma(u) / n, \ldots, \sigma(u) / n, \underline{0}) \succsim(\sigma(v) / n, \ldots, \sigma(v) / n, \underline{0}) .
$$

Using reflexivity of $\succsim$ if $\sigma(u)=\sigma(v)$ and SP on $[0,1]^{\mathbb{N}} \cap \mathscr{U}_{0}$ otherwise, this is equivalent with $\sigma(u) \geq \sigma(v)$.

(c): That a SWR ordering summable streams in $[0,1]^{\mathbb{N}}$ according to their sums satisfies the stated axioms follows as before. For the converse, assume $\succsim$ satisfies the axioms. Take $u, v \in[0,1]^{\mathbb{N}} \cap \mathscr{U}_{\text {fin }}$ with $\sum_{n=1}^{\infty} u_{n} \geq \sum_{n=1}^{\infty} v_{n}$. Since $u \in \mathscr{U}_{\text {fin }}$, $u_{i}<1$ for infinitely many $i \in \mathbb{N}$. Choose an $i>1$ with $u_{i}<1$. Let $\tilde{u} \in[0,1]^{\mathbb{N}}$ be obtained from $u$ by permuting coordinates 1 and $i$. By FA on $[0,1]^{\mathbb{N}} \cap \mathscr{U}_{\text {fin }}$,

\footnotetext{
${ }^{4}$ Likewise, Theorem 6 shows that the requirement $u_{n}=v_{n}$ for all large $n$ can be weakened in the characterization of $\succsim U$.
} 
$\tilde{u} \sim u$, so it suffices to show that $\tilde{u} \succsim v$. Evidently, $\sum_{n=1}^{\infty} \tilde{u}_{n}=\sum_{n=1}^{\infty} u_{n}$. By construction, $0 \leq \tilde{u}_{1}<1$. Let $\varepsilon>0$ be such that $\tilde{u}+(\varepsilon, \underline{0}) \in[0,1]^{\mathbb{N}}$. From this point onward, we can reason as in the corresponding part of the proof of Theorem 1 with $\tilde{u}$ instead of $u$, to show that $\tilde{u} \succsim v$. To be sure, $\tilde{u}+(\varepsilon, \underline{0}) \in[0,1]^{\mathbb{N}}$ implies that $\tilde{u}+(\varepsilon / 2, \underline{0}) \in[0,1]^{\mathbb{N}}$. By convergence of the two series there is an $N \in \mathbb{N}$ with $\sum_{i=1}^{n} \tilde{u}_{i}+\frac{\varepsilon}{2}>\sum_{i=1}^{n} v_{i}$ for all $n \geq N$. Equivalently, for all $n \geq N$, streams $\tilde{u}_{[n]}+(\varepsilon / 2, \underline{0})$ and $v_{[n]}$ in $[0,1]^{\mathbb{N}} \cap \mathscr{U}_{0}$ satisfy $\sigma\left(\tilde{u}_{[n]}+(\varepsilon / 2, \underline{0})\right)>\sigma\left(v_{[n]}\right)$. By part (b), $\tilde{u}_{[n]}+(\varepsilon / 2, \underline{0}) \succ v_{[n]}$ for all $n \geq N$. By HC on $[0,1]^{\mathbb{N} \cap} \mathscr{U}_{\text {fin }}, \tilde{u}+(\varepsilon / 2, \underline{0}) \succsim v$. By $\operatorname{SP}$ on $[0,1]^{\mathbb{N}} \cap \mathscr{U}_{\text {fin }}, \tilde{u}+(\varepsilon, \underline{0}) \succ \tilde{u}+(\varepsilon / 2, \underline{0})$. By transitivity, $\tilde{u}+(\varepsilon, \underline{0}) \succ v$. Since our $\varepsilon>0$ with $\tilde{u}+(\varepsilon, \underline{0}) \in[0,1]^{\mathbb{N}}$ was arbitrary, $\varepsilon \mathrm{C}$ on $[0,1]^{\mathbb{N}} \cap \mathscr{U}_{\text {fin }}$ gives $\tilde{u} \succsim v$.

Let $u, v \in[0,1]^{\mathbb{N}}$ be summable with $u \succsim v$. To show: $\sum_{n=1}^{\infty} u_{n} \geq \sum_{n=1}^{\infty} v_{n}$. If, to the contrary, $\sum_{n=1}^{\infty} u_{n}<\sum_{n=1}^{\infty} v_{n}$, then $v_{i}>0$ for some $i \in \mathbb{N}$. Hence, for $\varepsilon \in\left(0, v_{i}\right)$ sufficiently small, $v-\varepsilon e_{i} \in[0,1]^{\mathbb{N}}$ is summable and $\sum_{n=1}^{\infty} v_{n}-\varepsilon \geq \sum_{n=1}^{\infty} u_{n}$. Applying part (b) to summable streams $v-\varepsilon e_{i}$ and $u$ gives $v-\varepsilon e_{i} \succsim u$. By SP on $[0,1]^{\mathbb{N}} \cap \mathscr{U}_{\text {fin }}, v \succ v-\varepsilon e_{i}$. By transitivity, $v \succ u$, contradicting $u \succsim v$.

By restricting the SWRs in section 4.2 to $[0,1]^{\mathbb{N}}$, we find that the properties in Theorem 4 are logically independent.

4.4. Distinct averages. Imposing some of our axioms on the set $\mathscr{U}_{\mathrm{av}}$ of streams with well-defined, finite averages allows us to discriminate among streams with distinct averages. Observe that our continuity requirement $\varepsilon \mathrm{C}$ is not required here.

Theorem 5. If a $S W R \succsim$ on $\mathscr{U}$ satisfies $\mathrm{FA}\left(\mathscr{U}_{0}\right), \mathrm{FC}\left(\mathscr{U}_{0}\right), \operatorname{SP}\left(\mathscr{U}_{\mathrm{av}}\right)$ and $\mathrm{HC}\left(\mathscr{U}_{\mathrm{av}}\right)$, then $u \succ v$ for all $u, v \in \mathscr{U}_{\mathrm{av}}$ with $\bar{u}>\bar{v}$.

Proof. Take $u, v \in \mathscr{U}_{\text {av }}$ with $\bar{u}>\bar{v}$. Then there are $c>0$ and $N \in \mathbb{N}$ with $\frac{1}{n} \sum_{i=1}^{n} u_{i}-c>\frac{1}{n} \sum_{i=1}^{n} v_{i}$ for all $n \geq N$. Hence, $\sum_{i=1}^{n} u_{i}-c>\sum_{i=1}^{n} v_{i}$ for all $n \geq N$. Equivalently: for all $n \geq N$, the streams $u_{[n]}-(c, \underline{0})$ and $v_{[n]}$ in $\mathscr{U}_{0}$ satisfy

$$
\sigma\left(u_{[n]}-(c, \underline{0})\right)=\sum_{i=1}^{n} u_{i}-c>\sum_{i=1}^{n} v_{i}=\sigma\left(v_{[n]}\right) .
$$

Lemma 1 (b), which applies since $\mathrm{SP}$ on $\mathscr{U}_{\text {av }}$ implies $\mathrm{SP}$ on $\mathscr{U}_{0}$, gives $u_{[n]}-(c, \underline{0}) \succ$ $v_{[n]}$ for all $n \geq N$. By horizon consistency on $\mathscr{U}_{\mathrm{av}}, u-(c, \underline{0}) \succsim v$. By SP on $\mathscr{U}_{\mathrm{av}}$, $u \succ u-(c, \underline{0})$. By transitivity, $u \succ v$.

With minor changes in the axioms, requiring $\mathrm{HC}$ on pairs of streams $u, v$ with $u-v \in \mathscr{U}_{\text {av }}$ and SP on $\mathscr{U}$, the theorem can be extended to streams whose difference belongs to $\mathscr{U}_{\mathrm{av}}$.

\section{A characterization of the Utilitarian SWR}

Basu and Mitra [4, Theorem 1] characterize their utilitarian SWR $\succsim_{U}$, defined in (5), as the weakest SWR satisfying SP, FA, and the Partial unit comparability axiom:

$$
\forall u, v, \alpha \in \mathscr{U} \text {, if } u \succsim v \text { and } u^{[n]}=v^{[n]} \text { for some } n \in \mathbb{N} \text {, then } u+\alpha \succsim v+\alpha .
$$

To be precise, their characterization is on the smaller domain $[0,1]^{\mathbb{N}}$, so their version of the axiom says that if $u \succsim v$ and $u^{[n]}=v^{[n]}$ for some $n \in \mathbb{N}$, then $u+\alpha \succsim v+\alpha$ for every $\alpha \in \mathbb{R}^{\mathbb{N}}$, provided that all streams are in $[0,1]^{\mathbb{N}}$; cf. [4, p. 354]. 
Theorem 6 provides a variant of their characterization in two respects: (1) we consider the less restrictive domain $\mathscr{U}$ instead of $[0,1]^{\mathbb{N}} ;(2)$ our unit comparability axiom does not impose restrictions on all streams $u, v$ that are eventually identical, but only on streams that are eventually zero. That is, we require only:

$$
\forall u, v \in \mathscr{U}_{0} \text {, if } u \succsim v \text {, then } u+\alpha \succsim v+\alpha \text { for every } \alpha \in \mathscr{U},
$$

which is precisely Unit Comparability on $\mathscr{U}_{0}$. A by-product of Theorem 6 is the equivalence of the Partial unit comparability axiom and $\mathrm{UC}\left(\mathscr{U}_{0}\right)$ for ethical SWRs.

Theorem 6. A SWR on $\mathscr{U}$ is an extension of $\succsim_{U}$ if and only if it satisfies FA, $\mathrm{SP}$ and $\mathrm{UC}\left(\mathscr{U}_{0}\right)$.

Proof. Any extension of $\succsim_{U}$ satisfies SP, FA and (13) by an adaptation of [4, Theorem 1] to the domain $\mathscr{U}$. Since (13) implies (14), UC( $\left.\mathscr{U}_{0}\right)$ must hold as well. Conversely, let $\succsim$ be a SWR that satisfies $\mathrm{SP}, \mathrm{FA}$ and $\mathrm{UC}\left(\mathscr{U}_{0}\right)$.

Let $u, v \in \mathscr{U}$ satisfy $u \succsim U v$. To show: $u \succsim v$.

By definition of $\succsim_{U}$, there is an $N \geq 2$ with $u^{[N]} \geq v^{[N]}$ and $\sum_{i=1}^{N}\left(u_{i}-v_{i}\right) \geq 0$. Then $\sigma\left(u_{[N]}\right) \geq \sigma\left(v_{[N]}\right)$, so $\left(\sigma\left(u_{[N]}\right), \underline{0}\right) \succsim\left(\sigma\left(v_{[N]}\right), \underline{0}\right)$ by reflexiveness and SP. By Lemma 1 (a), which applies since $\mathrm{UC}\left(\mathscr{U}_{0}\right)$ implies $\mathrm{FC}\left(\mathscr{U}_{0}\right), u_{[N]} \succsim v_{[N]}$. Then (14) with $u_{[N]}, v_{[N]} \in \mathscr{U}_{0}$ and $\alpha=u^{[N]} \in \mathscr{U}$ gives $u \succsim\left(v_{1}, \ldots, v_{N}, u^{[N]}\right)$, hence $u \succsim v$ since $\left(v_{1}, \ldots, v_{N}, u^{[N]}\right) \geq v$.

Let $u, v \in \mathscr{U}$ satisfy $u \succ_{U} v$. To show: $u \succ v$.

We claim that there is an $N \geq 2$ with $u^{[N]} \geq v^{[N]}$ and $\sum_{i=1}^{N}\left(u_{i}-v_{i}\right)>0$. As in the previous case, there is an $N \geq 2$ with $u^{[N]} \geq v^{[N]}$ and $\sum_{i=1}^{N}\left(u_{i}-v_{i}\right) \geq 0$. If $u^{[N]}=v^{[N]}$, then $\sum_{i=1}^{N}\left(u_{i}-v_{i}\right)>0$, for $\sum_{i=1}^{N}\left(u_{i}-v_{i}\right)=0$ would imply $v \succsim_{U} u$. If $u^{[N]}>v^{[N]}$, let $N^{\prime}>N$ have $u_{N^{\prime}}>v_{N^{\prime}}$. Then $u^{\left[N^{\prime}\right]} \geq v^{\left[N^{\prime}\right]}$ and $\sum_{i=1}^{N^{\prime}}\left(u_{i}-v_{i}\right) \geq$ $\sum_{i=1}^{N}\left(u_{i}-v_{i}\right)+\left(u_{N^{\prime}}-v_{N^{\prime}}\right)>0$. Taking $c>0$ such that $\sigma\left(u_{[N]}\right) \geq \sigma\left(v_{[N]}\right)+c$ and repeating the above argument with $u$ and $v+(c, \underline{0})$ gives $u \succsim v+(c, \underline{0})$. It then follows by SP that $u \succ v$.

The corresponding result for SWRs on $[0,1]^{\mathbb{N}}$ is proved under the aforementioned modifications of the axioms.

\section{REFERENCES}

[1] K. Banerjee. On the extension of the utilitarian and Suppes-Sen social welfare relations to infinite utility streams. Social Choice and Welfare, 27:327-339, 2006.

[2] K. Banerjee and T. Mitra. On the continuity of ethical social welfare relations on infinite utility streams. Social Choice and Welfare, 30:1-12, 2008.

[3] K. Basu and T. Mitra. Aggregating infinite utility streams with intergenerational equity: the impossibility of being Paretian. Econometrica, 71, 2003.

[4] K. Basu and T. Mitra. Utilitarianism for infinite utility streams: A new welfare criterion and its axiomatic characterization. Journal of Economic Theory, 133:350-373, 2007.

[5] W. A. Brock. An axiomatic basis for the Ramsey-Weizsäcker overtaking criterion. Econometrica, 38:927-929, 1970.

[6] D. E. Campbell. Impossibility theorems and infinite horizon planning. Social Choice and Welfare, 2:283-293, 1985.

[7] C. d'Aspremont and L. Gevers. Equity and the informational basis of collective choice. Review of Economic Studies, 44:199-209, 1977.

[8] P. Fishburn and W. Edwards. Discount-neutral utility models for denumerable time streams. Theory and Decision, 43:139-166, 1997.

[9] D. Gale. On optimal development in a multi-sector economy. Review of Economic Studies, 34:1-18, 1967. 
[10] A. Jonsson and M. Voorneveld. The limit of discounted utilitarianism. SSE/EFI Working Paper Series in Economics and Finance, No. 748, 2014.

[11] L. Lauwers. Continuity and equity with infinite horizons. Social Choice and Welfare, 14:345356, 1997.

[12] L. Lauwers. Ordering infinite utility streams comes at the cost of a non-Ramsey set. Journal of Mathematical Economics, 46:32-37, 2010.

[13] E. Maskin. A theorem on utilitarianism. Review of Economic Studies, 45:93-96, 1978.

[14] J. Milnor. Games against nature. In R. Thrall, C. Coombs, and R. Davis, editors, Decision processes, chapter IV, pages 49-59. Chapman \& Hall, 1954

[15] H. Moulin. Axioms of cooperative decision making, volume 15 of Econometric Society Monographs. Cambridge University Press, 1988.

[16] A. Sen. Collective Choice and Social Welfare. Oliver \& Boyd, Edinburgh, 1971.

[17] P. Suppes. Some formal models of grading principles. Synthese, 16:284-306, 1966.

[18] L.-G. Svensson. Equity among generations. Econometrica, 48:1251-1256, 1980.

[19] C. C. von Weizsäcker. Existence of optimal programs of accumulation for an infinite time horizon. Review of Economic Studies, 32:85-104, 1965.

[20] P. Wakker. The repetitions approach to characterize cardinal utility. Theory and Decision, 7:33-40, 1986.

[21] W. Zame. Can intergenerational equity be operationalized? Theoretical Economics, 2:187$202,2007$.

Department of Engineering Sciences and Mathematics, Luleå University of TechnolOGY, 97187 LulEÅ, SWEdEN

E-mail address: adam.jonsson@ltu.se

Department of Economics, Stockholm School of Economics, 11383 Stockholm, SweDEN

E-mail address: mark.voorneveld@hhs.se 